\title{
Integrated curriculum based on projects: Opportunities and Constraints A Case Study in Systems Engineering
}

\author{
Sonia Mora, Mayela Coto \\ Escuela de Informática \\ Universidad Nacional \\ Heredia, Costa Rica \\ sonia.mora.rivera@una.cr; mcoto@una.cr
}

\begin{abstract}
The Universidad Nacional is making significant efforts to improve the professional performance of graduates according to the needs of the society and the industry. In particular, the School of Informatics has been exploring the opportunities to implement, through the use of a pedagogy oriented to problems and projects, a curricular integration between diverse areas of knowledge in the curriculum. From this perspective, an initial exploratory study was carried out, with the aim of integrating the areas of programming, databases and systems engineering. The findings show several limitations, such as mismatches between the course contents, lack of faculty commitment to collaborative work and student resistance. They also show the need of establishing strategies to create, from the early years of the curriculum, the necessary conditions to promote a positive attitude towards curricular integration processes and therefore overcome, at least partially, the identified limitations.
\end{abstract}

Keywords - curricular integration, integrative learning, pedagogical approaches, systems engineering curriculum

\section{INTRODUCCIÓN}

La integración curricular está relacionada con la forma en que se organizan los contenidos temáticos del currículo en actividades que propician la globalización del conocimiento [1]. Tiene como meta evitar la fragmentación de los aprendizajes y permitir que los estudiantes, ante una problemática real, puedan establecer relaciones entre diversos contenidos que le permitan proponer soluciones y lograr un aprendizaje significativo.

Cada vez con mayor frecuencia los problemas a resolver en cualquier entorno, ya sea gobierno, industria, o medicina son enfrentados y resueltos por grupos de personas de diferentes campos que deben trabajar juntas. Por ejemplo nuestro país como exportador de café, debe atender una enfermedad como la roya desde la perspectiva de varios especialistas: en cultivos, en enfermedades del café, y en desarrollo sostenible, que permitan encontrar soluciones efectivas que sean gentiles con los recursos naturales y los seres vivos. De esta manera el rol de la colaboración interdisciplinaria y el intercambio de experiencia y conocimiento se incrementa cada día [2].

Desafortunadamente muchos de los currículos universitarios se encuentran desarticulados, propiciando una serie de rupturas que se evidencian en el plano pedagógico, tales como repetición de áreas y temáticas en el desarrollo de los cursos, un conocimiento fragmentado, un esfuerzo doble para cumplir con las asignaciones de cada uno de los cursos, y pérdida de oportunidades para proporcionar a los estudiantes situaciones en las que pueden integrar el conocimiento de áreas disciplinarias para resolver un problema. Este es el caso del plan de estudios de la carrera de Ingeniería en Sistemas de Información de la Universidad Nacional de Costa Rica (UNA), donde cada cuerpo de conocimiento se ve como un listado de contenidos aislado sin considerar la interrelación necesaria con otros cursos de áreas afines del plan de estudios.

La estructura actual del plan de estudios, un enfoque pedagógico mayoritariamente tradicional, y la tendencia al individualismo de los docentes universitarios son algunas de las causas que provocan dificultades para lograr una adecuada integración que, como dice [3] facilite a los estudiantes la interconexión de los conocimientos existentes con los nuevos, las destrezas y la experiencia y así de esta manera puedan responder mejor a los cambios que la sociedad necesita.

Este artículo presenta una experiencia exploratoria de integración curricular entre tres áreas disciplinarias del plan de estudio de la carrera de Ingeniería en Sistemas informática de la Universidad Nacional: Programación, Bases de Datos e Ingeniería de Sistemas. El estudio tiene como meta analizar las oportunidades para que dichas áreas del conocimiento sean integradas en un solo proyecto y de esta forma lograr que el aprendizaje de los estudiantes sea más significado y relacionado con su entorno y su futura realidad profesional. En la primera parte del artículo se introducen los referentes 
teóricos relevantes, posteriormente se explica el contexto en el que se realiza el estudio, y la perspectiva docente y estudiantil con respecto al proceso de integración curricular. Finalmente se explican las limitaciones encontradas, posibles alternativas a las mismas y una reflexión final.

\section{REFERENTE TEÓRICO}

La experiencia universitaria es a menudo un conjunto de conocimientos y experiencias fragmentadas que no preparan a los estudiantes para enfrentarse al mundo real. Promover la capacidad del estudiante para integrar el aprendizaje adquirido a través de sus estudios, y entre la vida académica y personal es un reto importante de la educación superior [4] ya que como establece [5], la integración de los conocimientos y las perspectivas multidisciplinarias son requisitos de alta prioridad para los profesionales actuales.

En general, los académicos universitarios tienden a cubrir la mayor cantidad de contenidos en sus cursos, creando pocas oportunidades para que los estudiantes construyan soluciones y tomen decisiones alrededor de problemas complejos que involucran diversas áreas del conocimiento [2], es decir el conocimiento se ve como un ente encapsulado dentro de una misma especialidad y no como un cuerpo que requiere articulación con otros saberes.

La integración curricular es definida en [6] como "una acción que se orienta hacia la búsqueda de relaciones e interconexión entre los contenidos curriculares. Se trata de unir las partes a la totalidad, tal como se da en la realidad, sin que ello implique abandonar principios de orden, separabilidad y lógica". Es decir, la integración curricular es un enfoque que pretende superar la separación por cursos o asignaturas de las áreas del conocimiento, de manera que el aprendizaje sea funcional [1], y contribuya a que los estudiantes puedan darle un sentido conjunto a las piezas de conocimiento. En este sentido es importante aclarar que la integración curricular no es una sumatoria de materias. No se trata de que se unan contenidos de varias cursos y que sea el docente el responsable de realizar la conexión entre los conocimientos [7]. El aprendizaje integral debe desarrollar en los estudiantes estrategias que les permiten establecer por sí mismos nuevas relaciones entre contenidos diversos, generando así aprendizajes significativos en un espectro amplio de situaciones, lo que por ende los prepara mejor para su vida profesional [4] [1].

La integración curricular requiere trabajo. Los estudiantes juegan un papel fundamental, pero el éxito de las iniciativas depende en gran medida del compromiso y la creatividad de los docentes y de la administración [4]. A pesar de esto y aún cuando cada académico, individualmente puede contribuir en forma importante para fortalecer el aprendizaje integral, a través del diseño de su curso, del enfoque pedagógico utilizado y de las actividades de aprendizaje propuestas, [8] estos esfuerzos individuales, por sí mismos, no pueden darle sostenibilidad a una estrategia de integración curricular en una carrera universitaria. Para que esto suceda, es necesario un esfuerzo de colaboración conjunto entre todos los actores involucrados: autoridades, académicos y estudiantes.

Además, es vital que los líderes de la iniciativa realicen esfuerzos para lograr que la integración curricular sea de interés de toda la institución o departamentos involucrados; también se debe tener una estrategia clara de integración; contar con el apoyo de la administración; y tener claro que la integración es un proceso largo y gradual [4].

En [9] se identifican algunos de los obstáculos que enfrentan los procesos de integración curricular. Uno de ellos es la reacción ante nuevos enfoques. Como ante cualquier nueva iniciativa, hay académicos que no tienen disposición a comprometerse, ya sea por falta de interés o porque sienten que la experimentación puede perjudicar su trayectoria académica.

Otro obstáculo está relacionado con la dependencia de un liderazgo. Muchas veces los nuevos esfuerzos de integración curricular dependen de un único líder, y si este no tiene la energía o el poder requerido, los esfuerzos tienden a debilitarse. Para lograr procesos exitosos se requiere un liderazgo que tenga el poder, por ejemplo, de ofrecer a los académicos la posibilidad de que puedan asistir a reuniones y sesiones de capacitación. Además el o los líderes deben poder contribuir a construir relaciones positivas y efectivas entre los académicos participantes.

Entre otros de los obstáculos para una efectiva integración curricular se encuentran la carga de trabajo docente, la cobertura del plan de estudios, y la colaboración efectiva entre los docentes [9]. El incremento en la carga de trabajo de los docentes es uno de los mayores obstáculos, mientras algunos académicos están dispuestos a invertir más tiempo preparándose para abordar el enfoque, para otros no está dentro de sus prioridades. Adicionalmente, es difícil para las instituciones reconocer el tiempo o brindar los incentivos necesarios para incursionar en iniciativas de integración curricular. Otro inconveniente está relacionado con el plan de estudios, ya que existe el temor de que se logren cubrir menos contenidos debido a que deben enseñarse nuevas habilidades o al tiempo que consume el trabajo en grupo. En este sentido, los docentes pueden sentir que la integridad y el nivel de sus cursos se ven perjudicados. También algunos críticos de la integración afirman que la calidad de la educación puede disminuir al ser más aplicada que teórica [9].

Otros de los obstáculos surgen alrededor de la colaboración docente. Algunas dificultades pueden surgir cuando los docentes tienen diferentes perspectivas con respecto a los estudiantes, o diferentes estándares para evaluar un mismo trabajo (por ejemplo contenido vs claridad o calidad de la redacción). Esto último también puede causar confusión entre los estudiantes. 
Ante este escenario, es vital asegurar canales de comunicación abiertos y oportunos entre los docentes de los cursos o materias involucradas en cualquier proceso de integración curricular. Esta coordinación contribuye además a lograr que no se repitan contenidos y que estos más bien se complementen [8].

De acuerdo a [10], existen diferentes formas de integración curricular: tópico generador, integración por relato, integración por ejes temáticos e integración por proyectos:

- El tópico generador es una estrategia en la que se parte de la formulación de un problema común a diversas disciplinas o a diversas áreas del conocimiento que se articulan entre sí. El aspecto fundamental en esta estrategia es que para solucionar el problema propuesto se deben utilizar todas las disciplinas o campos del conocimiento involucrados [11].

- La integración por relato se realiza alrededor de un relato que articula los tópicos y problemas que desean plantearse de las diferentes disciplinas. Su objetivo principal es contribuir a contextualizar la problemática [12].

- La integración por ejes temáticos, se concreta a partir del acuerdo entre un grupo de docentes, de uno o varios ciclos, quienes en forma conjunta seleccionan un tema que debe ser abordado simultáneamente desde cada una de las áreas del conocimiento involucradas. Su principal objetivo es evidenciar que los conocimientos tienen aplicabilidad práctica en el entorno en el que se ubican los estudiantes [13]. En una versión avanzada de la estrategia, se propone que sean los estudiantes los que seleccionan los temas de acuerdo a sus intereses particulares.

- La integración por proyectos está relacionada con elementos como el aprendizaje activo, el trabajo colaborativo, la interacción docente-estudiante, la promoción de la autonomía en los estudiantes durante el proceso de construcción de conocimientos, el desarrollo de una capacidad creadora e investigativa, y un vínculo estrecho con la realidad como elemento para articular la teoría con la práctica [10]. Los proyectos constituyen una excelente opción de articulación horizontal entre temáticas o cursos de un mismo nivel.

Es claro, desde nuestra perspectiva, que las anteriores formas de integración, no son mutuamente excluyente ya que dependiendo del contexto una combinación de ellas puede enriquecer una situación específica de aprendizaje.

Además, según [14] el aprendizaje integrado está relacionado con hacer conexiones entre experiencias de aprendizaje, lo que ocurre desde la niñez y continúa a lo largo de la vida. A nivel universitario este proceso involucra: 1) la mezcla de destrezas y conocimientos de distintas disciplinas; 2) unir teoría y práctica; 3 ) considerar múltiples perspectivas para resolver problemas en grupos; 4) adaptar las destrezas aprendidas en una situación a otra; y por último 5) reflexionar sobre sus experiencias de aprendizaje y ser capaz de plasmarlas en un informe o ensayo.

El estudio que se plantea en este artículo utiliza como estrategia de integración curricular el enfoque por proyectos. Es decir se parte de una pedagogía por proyectos como el instrumento que permite generar la articulación de conocimientos entre cursos de un mismo nivel. Específicamente se utiliza el enfoque conocido como el Modelo PBL de Aalborg [15] o Project-Oriented Problem Pedagogy (POPP). El modelo está orientado a la resolución de problemas como base del proceso de inicio del aprendizaje. El problema se aborda mediante la realización de un proyecto que integre la teoría y la práctica. La búsqueda de soluciones viables al problema se realiza en forma colaborativa entre un grupo de estudiantes que reciben una continua realimentación de compañeros y docentes. Adicionalmente, los estudiantes tienen un nivel de autonomía en la selección del problema y en cómo organizar el proyecto, con lo que se pretende lograr un mayor compromiso y motivación hacia el aprendizaje [16].

Es claro que lo anterior implica dejar de lado la enseñanza tradicional y apegarse a un enfoque que promueve y estimula el aprendizaje colaborativo y centrado en el estudiante por medio de la solución a problemas de la vida real que integran, de forma natural, diversas áreas del conocimiento.

\section{DESCRIPCIÓN DEL CONTEXTO}

La Universidad Nacional cuenta desde el año 2008 con un modelo pedagógico que entiende la enseñanza y el aprendizaje como un proceso social, histórico y cultural que va más allá de la simple transmisión de conocimientos [17]. El modelo se basa en principios constructivistas donde se concibe al estudiante como el centro del proceso de aprendizaje. Además propone un aprendizaje activo y significativo a partir del entorno del estudiante. Sin embargo, como modelo, no señala ninguna estrategia didáctica específica, lo que conlleva a que se traduzca en las aulas de muy diversas maneras, dependiendo de factores tales como los diferentes objetos de estudio, la concepción que el docente tenga de la enseñanza y el aprendizaje, y sus habilidades para aplicar diferentes estrategias didácticas.

En el caso particular de la Escuela de Informática, la aplicación del modelo en los cursos depende, en muchos de los casos, de la naturaleza propia del curso y del conocimiento pedagógico que poseen los docentes que lo imparten. Para efectos de este artículo, nos interesa enfocarnos en los niveles quinto, sexto y sétimo de la carrera, y en la forma en que algunos de estos cursos organizan el proceso de aprendizaje. La Tabla 1 muestra los cursos que se imparten en dichos ciclos. 
TABLA 1. CURSOS DEL PLAN DE ESTUDIOS DE INGENIERÍA DE SISTEMAS

\begin{tabular}{|c|c|c|}
\hline Quinto ciclo & Sexto ciclo & Sétimo ciclo \\
\hline $\begin{array}{l}\text { Ingeniería de Sistemas } \\
\text { I }\end{array}$ & $\begin{array}{l}\text { Ingeniería de Sistemas } \\
\text { II }\end{array}$ & $\begin{array}{l}\text { Ingeniería de Sistemas } \\
\text { III }\end{array}$ \\
\hline Programación IV & $\begin{array}{l}\text { Paradigmas de } \\
\text { Programación }\end{array}$ & $\begin{array}{l}\text { Diseño y } \\
\text { programación de } \\
\text { plataformas móviles }\end{array}$ \\
\hline $\begin{array}{l}\text { Diseño e } \\
\text { Implementación de } \\
\text { Bases de Datos }\end{array}$ & $\begin{array}{l}\text { Administración de } \\
\text { Bases de Datos }\end{array}$ & $\begin{array}{l}\text { Métodos de } \\
\text { Investigación } \\
\text { Científica en } \\
\text { Informática }\end{array}$ \\
\hline Sistemas Operativos & $\begin{array}{l}\text { Investigación de } \\
\text { Operaciones y sus } \\
\text { Aplicaciones }\end{array}$ & Optativa \\
\hline $\begin{array}{l}\text { Comunicaciones y } \\
\text { Redes de } \\
\text { Computadoras }\end{array}$ & $\begin{array}{l}\text { Liderazgo y } \\
\text { Organización }\end{array}$ & Optativa \\
\hline
\end{tabular}

Fuente Plan de estudios de la Carrera Ingeniería en Sistemas de Información

Durante estos tres ciclos lectivos los estudiantes desarrollan de manera ininterrumpida un sistema de información en una empresa, utilizando un enfoque pedagógico orientado a la solución de problemas mediante proyectos, esto además de propiciar un aprendizaje significativo, les permite conocer la realidad nacional y descubrir su potencial como profesionales [18]. Este proceso es organizado y coordinado desde la secuencia de cursos de Ingeniería de Sistemas y los estudiantes son guiados por los profesores de dicha cátedra. Paralelamente los estudiantes están llevando, entre otros, cursos de Programación y Bases de Datos.

En los cursos de las tres áreas mencionadas, el proceso de aprendizaje gira fuertemente en torno a la realización de proyectos, sin embargo los mismos son de naturaleza muy diferente. En la cátedra de Ingeniería de Sistemas (IS) se trabaja con un único proyecto a través de los tres cursos: Ingeniería de Sistemas I, II y III. En estos cursos los estudiantes primero deben identificar una empresa que tenga una necesidad específica en el área de los sistemas de información, y a lo largo de los tres cursos desarrollan completamente la aplicación pasando por diferentes etapas guiadas por los docentes del curso. Desde el punto de vista de la coordinación académica, los docentes que participan en esta cátedra, están fuertemente organizados y tienen una alta cohesión, cada docente se "mueve" a través de los tres cursos para tener una mejor comprensión de los mismos. Además los estudiantes están organizados por grupos y cada uno tiene asociado un profesor titular. La cátedra, además unifica los temas y las entregas de productos intermedios. Por medio de un aula virtual se publica el material para todos los estudiantes de la cátedra, y todos los académicos utilizan el mismo material. El proyecto tiene el mayor peso entre las actividades de aprendizaje de los cursos, variando entre un 50\% y $60 \%$ [18].

Por su parte, en la cátedra de Programación se plantean proyectos muy puntuales que inician y acaban en cada uno de los cursos, dado que cada uno de ellos persigue objetivos y cubre temáticas muy específicos. Con respecto a la coordinación académica, ha sido muy difícil lograr una planificación y organización vertical al nivel de cátedra. A nivel horizontal si se tiene, en la mayoría de los casos, una buena coordinación a nivel de los profesores de un mismo curso, donde se comparten materiales y se planifican las mismas evaluaciones y tipos de proyectos. Se han hecho esfuerzos para que los materiales utilizados por los docentes en sus clases sean los mismos para todos los grupos de un mismo curso. Los proyectos generalmente representan de un $20 \%$ a un $30 \%$ del total de actividades de aprendizaje del curso.

La cátedra de Bases de Datos contempló inicialmente la realización de un proyecto específico en una empresa, sin embargo la implementación de esta propuesta no fue real por razones de la carga académica que los estudiantes enfrentaban en estos ciclos lectivos. Por esta razón se dejó de considerar esta posibilidad y actualmente los estudiantes realizan pequeños proyectos o trabajos que no les demandan tanta dedicación. Desde el punto de vista de coordinación, en cada uno de los cursos se planifican y organizan los mismos proyectos y actividades de evaluación, aunque cada docente prepara y utiliza sus propios materiales de clases.

Lo anterior muestra que cada una de las cátedras de curso tiene a lo interno niveles diferentes de organización, y de cohesión.

Como puede verse en la Tabla 1, la carga académica de los estudiantes durante estos tres ciclos es muy pesada, en un momento dado los estudiantes pueden estar desarrollando tres proyectos de naturaleza muy diferente. Adicionalmente los estudiantes se quejan de la poca interrelación que existe entre los académicos que imparten los cursos en un mismo nivel. Lo anterior no les parece congruente, dado que al ser el proyecto de los cursos de Ingeniería de Sistemas un proyecto integral que debe ser desarrollado en su totalidad, requiere sin lugar a dudas de componentes fuertes de programación y bases de datos. Actualmente, los estudiantes reciben muy poco apoyo en éstas áreas, ya que los docentes de la cátedra de Ingeniería no son expertos en las mismas, y al no existir coordinación con los docentes de Bases de Datos y Programación, la colaboración de los mismos al desarrollo del proyecto, si la hay, es muy tangencial. En efecto, cada docente está enfocado en el desarrollo de los proyectos dentro de su propio curso, de esta manera se desaprovecha la oportunidad no sólo de aligerar la carga de trabajo de los estudiantes sino de que trabajen el proyecto de manera integrada y con el apoyo de las distintas áreas disciplinarias involucradas.

Dado el contexto anterior, a partir del análisis de la literatura y de experiencias exitosas en otros países, y tomando como base el hecho de que ya la cátedra de Ingeniería de Sistemas incorpora en su práctica docente algunos principios del POPP [18], se consideró importante realizar un estudio exploratorio que determinara la viabilidad de utilizar el enfoque POPP como el medio que permite lograr una integración entre los cursos mencionados. Dada la natural interrelación entre las tres áreas del conocimiento, se 
consideró que estos niveles reunían las mejores condiciones para realizar una primera iniciativa de integración curricular incrementando la posibilidad de obtener experiencias positivas, ya que como [19] sugiere un elemento vital para lograr cambios significativos en las actitudes y prácticas de los docentes es que estos hayan tenido experiencias positivas relativas a la mejora del aprendizaje de sus estudiantes. Estas mejoras son generalmente el resultado de cambios que los docentes han hecho en el aula. En este sentido, el estudio propuesto sustenta el cambio como un proceso basado en experiencias de aprendizaje positivas para docentes y estudiantes.

El proceso de integración inicial se propone a nivel de la realización de un único proyecto que refleje adecuadamente la interrelación entre las tres áreas del conocimiento (programación, bases de datos e ingeniería de sistemas), y donde cada uno de los académicos aporte, desde su especialidad, el conocimiento y el apoyo que los estudiantes requieren para una realización exitosa del mismo.

La filosofía de la iniciativa propuesta parte de que son los mismos docentes especialistas en sus áreas y conocedores de los objetivos de cada uno de los cursos, los que pueden sugerir el tipo y el nivel de integración factible. Debido a esto se utilizó una metodología participativa con los académicos como actores principales. Se plantea un estudio cualitativo ya que lo que se pretende es lograr una mejor comprensión del comportamiento actual de las cátedras involucradas, las perspectivas que tanto académicos como docentes poseen al respecto de la integración curricular y las condiciones actuales de la Escuela de Informática para este tipo de iniciativas.

Partiendo de la premisa de que el proceso de integración es un proceso gradual que lleva tiempo, se visualizó un proceso exploratorio con las siguientes etapas: (1) sensibilización docente; (2) análisis de los posibles puntos de confluencia entre los cursos a nivel de objetivos, contenidos, metodología, didáctica y actividades de aprendizaje; (3) elaboración de una propuesta piloto que oriente la implementación y puesta en práctica de la integración en el nivel 5 de la carrera; (4) seguimiento/valoración a los estudiantes del nivel 5 en el desarrollo del proyecto integrado; y (5) análisis retrospectivo de las experiencias obtenidas en el 5to nivel. A partir de aquí las actividades se vuelven a repetir consecutivamente para los niveles 6 y 7 del plan de estudios, tomando como insumos los resultados obtenidos en el nivel anterior. Todo este proceso se acompaña de un plan de capacitación que les permita a los docentes desarrollar las competencias adecuadas para implementar los procesos de integración. Esto es acorde con [20] que sugiere que cuando los docentes se apropian de los cambios propuestos es más probable que los mismos sean sostenibles en el tiempo.

Como parte de estos procesos se consideró necesario conocer tanto la perspectiva docente como la estudiantil ante la posibilidad de la integración y a partir de ambas poder valorar adecuadamente el contexto en el que se enmarca la iniciativa, así como cuáles son, desde su perspectiva, las limitaciones o dificultades que se podrían enfrentar. En la siguiente sección se presentan los resultados obtenidos.

\section{A. Perspectiva docente}

Dentro de la etapa de sensibilización, de julio a octubre del 2011, se realizan un conjunto de sesiones de trabajo con académicos de las distintas cátedras: Programación, Bases de Datos e Ingeniería de Sistemas, para dar a conocer los fundamentos del enfoque POPP, y lograr una mayor comprensión sobre algunos aspectos fundamentales, tales como la forma en que cada área concibe y planifica los proyectos, los objetivos de aprendizaje de los mismos, y la disposición de los académicos para planificar un solo proyecto conjunto que integrara de forma efectiva los conocimientos de los tres cursos involucrados en cada uno de los ciclos quinto, sexto, y sétimo de la carrera.

Para conocer su perspectiva, los docentes involucrados en los ciclos lectivos en estudio fueron invitados a un taller. Se inició con algunas actividades motivadoras que propiciaban el intercambio y la comunicación asertiva, y posteriormente se dividió al grupo de ocho participantes en tres subgrupos, donde el primer grupo tenía como objetivo identificar cuáles eran las limitaciones que ellos visualizaban, el segundo grupo trabajaba sobre las actividades que permitieran disminuir estas limitaciones y el tercer grupo reflexionaba sobre las metas a obtener con el proceso de cambio.

El primer grupo de trabajo identificó una serie de barreras para realizar la integración curricular, las mismas giran alrededor de tres aspectos: la administración, el cuerpo docente y los estudiantes. Con respecto a la administración, los profesores señalan obstáculos en aspectos administrativos y curriculares, en un clima laboral de escasa motivación, y a la forma actual en que se conciben y realizan los diversos proyectos en cada curso. Con respecto al cuerpo docente, consideran que la mayoría de ellos posee esquemas mentales rígidos, donde no se concibe la pedagogía como una forma de mejorar el aprendizaje y la docencia; además expresan que su trabajo no es valorado adecuadamente y que existe escasa supervisión sobre la realización del mismo. Adicionalmente ven difícil colaborar y trabajar juntos. Finalmente, con respecto al estudiante, apuntan como barreras su bajo nivel de compromiso y motivación, así como su débil capacidad de abstracción y redacción.

El segundo grupo de profesores trabajó sobre algunos de los objetivos que les interesaba alcanzar a través de la integración curricular y en general sus intereses giraron alrededor de buscar un ambiente de trabajo más gratificante, donde se promueva una cultura de compartir y de aprender.

El último grupo trabajó en aquellos aspectos que reducen la brecha entra barreras y metas. Entre las acciones sugeridas se encuentran: diseñar una estrategia que promueva un proceso 
gradual de cambios; tener la mente abierta para cambios curriculares, y mejorar las relaciones y procesos de construcción del conocimiento entre profesores y estudiantes.

Posteriormente a este proceso, y teniendo mayor claridad sobre las intenciones y temores de los académicos involucrados, se realizan una serie de reuniones con los docentes de las cátedras para analizar los posibles puntos de confluencia entre los cursos, tanto a nivel de objetivos de aprendizaje como de contenidos y metodología. En estas sesiones se sigue evidenciando que los académicos tienen algunas inquietudes para el trabajo colaborativo, además se muestran recelosos de exponer los cursos que consideran de "su propiedad" a otros colegas. Cada cátedra se considera experta en su materia, la mayoría de ellos expresa, que si bien sus cursos son susceptibles a mejora, la forma en la que los imparten es adecuada y alcanza los objetivos de aprendizaje que se han fijado. Como puede verse, esta actitud es no congruente al deseo que los académicos expresaron en el primer taller sobre promover una cultura de compartir y de aprender.

De igual manera, los docentes apuntan a desfases importantes entre los contenidos de los cursos de las diferentes áreas en un nivel dado. A su criterio, estos desfases contribuyen a que, en las condiciones actuales del plan de estudio, los cursos no puedan complementarse de manera efectiva para apoyar la realización de un único proyecto integrado.

Además se detectan diferentes niveles de comprensión y disposición hacia el enfoque POPP. La cátedra de Ingeniería de Sistemas, argumenta tener experiencia en la implementación de algunos de los principios del POPP, y haber realizado adelantos significativos en la organización y planificación requeridos por el enfoque. Para las cátedras de Programación y Bases de Datos, el enfoque POPP a nivel de organización del proceso de aprendizaje, es algo totalmente nuevo, aunque si están familiarizadas con la realización de proyectos en sus cursos. Adicionalmente, para la cátedra de Ingeniería de Sistemas, el proyecto que realizan durante los tres ciclos lectivos demanda una gran cantidad de esfuerzo y planificación entre todos los docentes de la cátedra. Involucrarse en una posible integración con cursos de otras áreas significa una mayor cantidad de trabajo, así como la posibilidad de replantearse temas, tiempos y tareas conjuntas. Lo anterior podría tener el efecto contraproducente de detener o incluso retroceder en los avances obtenidos por ellos hasta la fecha.

Después de varias sesiones adicionales donde los temas de discusión se vuelven recurrentes y prácticamente no se logra ningún avance en el camino deseado, los académicos hacen notar que no se cuenta con el tiempo, a nivel de las cátedras de la Escuela, para planificar ni coordinar la realización de un único proyecto en el que se integre las tres áreas disciplinarias. Según su criterio, esto requiere mucho tiempo y un esquema de coordinación conjunta, difícil de realizar en las condiciones actuales del plan de estudios y de la Escuela.

En este sentido, los docentes, sugieren primero realizar cambios paulatinos a lo interno de las diferentes áreas del conocimiento antes de pensar en integrarlas.

\section{B. Perspetiva estudiantil}

Con respecto a la perspectiva estudiantil se consideraron 68 estudiantes del cuarto nivel de la carrera. Esta población estudiantil tiene experiencia en el desarrollo de proyectos, que por lo general tratan sobre problemas ficticios que simulan situaciones de la vida real. Además es la población que está más próxima a entrar a la secuencia de cursos de Ingeniería de Sistemas, donde se dan las mejores condiciones para realizar un proyecto orientado a la solución de problemas auténticos. Debido al nivel de la carrera en la que se encuentran, se consideró que la opinión de estos estudiantes permitiría analizar su disposición hacia la integración curricular por medio de proyectos. Para tales efectos, se elaboró un cuestionario auto-administrado, compuesto de preguntas abiertas y cerradas.

La Tabla 2 muestra tres de los aspectos valorados. Como puede verse la mayoría de los estudiantes (79\%) piensa que los contenidos de los cursos se ven reflejados adecuadamente en los proyectos. Adicionalmente cuando se les pregunta sobre si los proyectos de los cursos integran las distintas áreas disciplinarias, un $72 \%$ responde afirmativamente. Sin embargo, únicamente un $46 \%$ muestra una actitud positiva hacia la integración de los contenidos de varios cursos del mismo nivel en un único proyecto.

TABLA 2. VALORACIÓN DE ASPECTOS RELACIONADOS A LA

\begin{tabular}{|c|c|c|c|c|}
\hline Aspecto a considerar & SI & $\%$ & NO & $\%$ \\
\hline $\begin{array}{l}\text { 1. ¿Considera usted que en los } \\
\text { cursos de la carrera los contenidos } \\
\text { teóricos se ven reflejados en la } \\
\text { realización de los proyectos de los } \\
\text { cursos? }\end{array}$ & 54 & 0,79 & 14 & 0,21 \\
\hline $\begin{array}{ll}2 . & \text { ¿Considera usted que los } \\
\text { proyectos de los cursos llegan a } \\
\text { integrar las distintas áreas } \\
\text { disciplinarias de la carrera? }\end{array}$ & 49 & 0,72 & 19 & 0,28 \\
\hline $\begin{array}{l}\text { 3. ¿Le gustaría que los cursos de la } \\
\text { carrera que se encuentren en un } \\
\text { mismo nivel, pudieran integrar los } \\
\text { aspectos teórico/prácticos en un } \\
\text { sólo proyecto? }\end{array}$ & 31 & 0,46 & 37 & 0,54 \\
\hline
\end{tabular}

Cuando se les pide a los estudiantes que razonen su respuesta, se identifica que aquellos que están a favor de la integración en un único proyecto, dan como razones principales: (1) un mejor balance de la carga académica ya que en lugar de distribuir el tiempo entre tres proyectos diferentes pueden enfocarse a la realización de uno solo; (2) mejor integración del conocimiento lo que les permite tener una visión más global y real de los problemas. Por otro lado, los 
estudiantes que no están de acuerdo con esta propuesta, expresan preocupaciones de índole práctico como que (1) no todos los estudiantes matriculan los tres cursos al mismo tiempo; (2) les preocupa que el proyecto tenga mayor nivel de complejidad y como tal sea más difícil de resolver; (3) que un fallo en el proyecto afectaría negativamente la evaluación de todos los cursos involucrados. Por otro lado también les preocupa la capacidad de organización y de búsqueda de consensos de los docentes de los cursos involucrados.

Es interesante notar que los resultados obtenidos en las preguntas 2 y 3 parecen contradictorios. Sin embargo, es posible que los estudiantes no tengan pleno conocimiento de lo que implica la integración de las áreas disciplinarias en un solo proyecto, así como también que los temores de los estudiantes claramente no están asociados a la integración de distintas áreas del conocimiento en un único proyecto, sino más bien a las consecuencias que podría tener en su evaluación que tres cursos basaran su calificación sobre un mismo producto. Es claro, como los mismos estudiantes lo señalan, que un proceso como estos requiere de una gran labor de coordinación entre los académicos involucrados.

De lo anterior puede deducirse, que antes de realizar iniciativas de integración curricular debe hacerse un trabajo de sensibilización con los estudiantes, probablemente desde su primer año en la universidad, donde se valoren más las oportunidades de un aprendizaje integrado y se les brinden herramientas para poder enfrentar las limitaciones o problemas que ellos detectan en el proceso. Paralelo a esto, es claro que el mismo proceso debe realizarse con los académicos, para lograr cambios positivos hacia los procesos de integración. De forma particular, estos procesos deben venir acompañados de un apoyo de las autoridades de la Escuela de Informática, que son los que pueden establecer las líneas de acción a seguir a nivel Escuela.

\section{LIMITACIONES IDENTIFICADAS}

Como resultado de estos procesos de consulta y trabajo conjunto con profesores y estudiantes se logra determinar que actualmente no se tienen las condiciones, a nivel administrativo ni a nivel docente y estudiantil, para lograr una integración efectiva de los cursos en los niveles quinto, sexto y sétimo de la carrera de Ingeniería en Sistemas de Información. Las limitaciones encontradas se sustentan principalmente en la organización actual del plan de estudio y en la carencia de una sólida cultura de colaboración, como se explica a continuación:

\section{A. Organización actual del plan de estudios}

Al proponer la realización de un sólo proyecto que integre al menos tres de los cursos que se imparten en un nivel de la carrera, la formulación del mismo debe surgir de un proceso de trabajo conjunto y colaborativo entre los docentes que imparten los cursos, y debe permitir que cada una de las áreas disciplinarias involucradas logre alcanzar sus propios objetivos de aprendizaje.

Como puede verse, en la Tabla 1, el quinto nivel de la carrera reúne las condiciones ideales para la integración (se imparten los cursos de las tres áreas). Sin embargo, en el curso de Ingeniería de Sistemas I, los estudiantes realizan actividades de análisis de requerimientos y diseño, que no requieren de ninguna labor de programación ni del diseño de bases de datos, de esta manera los conocimientos no los adquieren en el momento oportuno para aplicarlo en el curso de Ingeniería de Sistemas. Esto genera un desfase de contenidos. Una situación igual se presenta en el sexto nivel, de nuevo y aunque en este ciclo lectivo se imparten cursos de las tres áreas, las temáticas cubiertas por cada curso no están relacionadas entre sí, ni tienen como objetivo servir a un fin común: diseño y desarrollo de una aplicación informática y finalmente, en el sétimo nivel de la carrera ya no llevan cursos de bases de datos, y es cuando los estudiantes del curso de Ingeniería de Sistemas III finalizan con el desarrollo del sistema y requieren más apoyo en esas áreas.

De tal manera en este primer acercamiento, los docentes de cada área determinaron que sus contenidos y objetivos de aprendizaje son muy concretos y puntuales, y que se encuentran diferidos en el tiempo (en los contenidos de los cursos correspondientes del plan de estudios) y que tratar de hacer un proyecto que integre los conocimientos requeridos para la implementación adecuada del mismo, puede poner en peligro el logro de los objetivos y contenidos de cada curso en sí mismo.

De lo anterior puede deducirse que según los niveles de la carrera y los contenidos actuales de los cursos no es conveniente en este momento adecuarlos para coordinar las temáticas de los tres cursos al desarrollo de un único proyecto, desde la perspectiva de la Ingeniería de Sistemas, sin afectar el plan de estudios.

\section{B. Cultura colaborativa}

Por último, pero no menos importante es que hay que tener muy claro que la integración de cursos demanda esfuerzo, compromiso, trabajo adicional y mucha organización $\mathrm{y}$ planificación de parte de los académicos de las cátedras involucradas, que son los actores fundamentales del proceso de integración curricular [4].

Sin embargo, al analizar las posibilidades existentes, no se encuentra en este momento un ambiente de confianza, compañerismo, colaboración y apertura entre las cátedras involucradas en esta iniciativa. Se evidencian problemas de "islas" ya no a nivel de individuos pero si de las mismas cátedras, que no abren espacios de trabajo conjunto con otras áreas del conocimiento. Además, y en forma consistente con lo señalado por [9], las múltiples ocupaciones de los docentes les 
impiden participar de un proceso de capacitación que propicie un ambiente colaborativo hacia la integración de las distintas áreas del conocimiento del plan de estudios.

\section{POSIBLES ALTERNATIVAS}

A pesar de que el estudio indica limitaciones, semejantes a las identificadas por [9], que no hacen posible la iniciativa de integración curricular mediante un proyecto único en las tres áreas del conocimiento mencionadas, se considera que las oportunidades siguen existiendo, y que es viable realizar acciones que vayan reduciendo el alcance de las limitaciones.

Con respecto al desfase de contenidos en los cursos es posible plantear un rediseño parcial del currículo, cambiando algunos cursos de nivel, sin que eso signifique un rediseño completo del plan de estudios. Se modifica el nivel en que se imparten algunos de los cursos, y se hace un ajuste de contenidos en los cursos de programación y bases de datos. Un enfoque más agresivo es pensar en un rediseño completo del plan de estudios de la carrera considerando la integración curricular como eje central al plan de estudios, y tomando el enfoque pedagógico POPP como el medio para lograr la integración. En esta línea se podría usar una propuesta similar a la de la Universidad de Aalborg, con cursos de proyectos y cursos teóricos complementarios que se integran a través de un tema o tópico generador, como lo señala [11], claramente identificado por ciclo lectivo.

En relación a la poca apertura de las cátedras a trabajar conjuntamente con otras áreas del conocimiento, se visualiza necesario provocar un cambio de actitud. Esto puede lograrse a través de un proceso de conformación de equipos de trabajo siguiendo las recomendaciones de [21] que establece la necesidad de conocer mejor a los participantes, de plantear procesos que les permitan tener mayor claridad acerca de lo que se pretende lograr, y generar un ambiente de trabajo conjunto con el objetivo de promover metas de trabajo comunes, de tal manera que los docentes participantes puedan apropiarse de la iniciativa y tener mayor apertura hacia los ajustes necesarios en los procesos de integración curricular. Adicionalmente, como señala [9], es importante la identificación de líderes que puedan promover la motivación y compromiso de sus compañeros de cátedra. Todo esto debe ser acompañado de un apoyo institucional bien definido.

Con respecto a la resistencia de los estudiantes, es importante proporcionarles experiencias de aprendizaje que les permitan reconocer el valor de lograr una articulación de áreas del conocimiento, de integrar teoría y práctica, de resolver problemas y trabajar en equipos, considerando adecuadamente aspectos como el manejo de la carga académica, y la resolución de conflictos.

\section{REFLEXIONES FINALES}

Es importante entender que la integración curricular puede permitir construir conocimientos significativos, pertinentes y adecuados a las necesidades y demandas actuales de los profesionales, pero que deben ser diseñadas a futuro las estrategias que permitan a los docentes, como primeros responsables del trabajo en el aula la construcción de estos currículos integrados.

En el proceso exploratorio se determina que la integración inmediata de los cursos de los niveles quinto, sexto y sétimo de la carrera de Ingeniería en Sistemas de Información no es viable. Los resultados obtenidos en las etapas de sensibilización y de análisis de puntos de confluencia, muestran que el plan de estudios no facilita la integración de contenidos, que los académicos aún no están listos para el trabajo colaborativo, y que hace falta apertura hacia el trabajo con otras áreas del conocimiento ajenas a su especialidad.

Es evidente la necesidad de crear los espacios a nivel de unidad académica que permita mostrar a los docentes la importancia de la integración de contenidos y áreas disciplinarias en la formación de los estudiantes, y que les permitan tener una visión más amplia de currículo, más allá de los cursos de su especialidad. Pareciera que este proceso debe iniciar a lo interno de cada una de las áreas construyendo confianza entre los académicos, y posteriormente proyectarse hacia el trabajo entre las distintas áreas del conocimiento.

Este primer intento logra identificar las limitaciones existentes, pero también muestra el potencial del enfoque POPP para facilitar la integración curricular. Si dicho enfoque se adopta desde los primeros niveles del plan de estudios [22], se irán construyendo de manera natural la sinergia necesaria entre los académicos y se irán desarrollando entre los estudiantes las habilidades requeridas para el abordaje y solución de problemas complejos de la vida real.

Por último, hay que considerar que para que el aprendizaje integral sea realmente efectivo no debe ser un evento independiente sino una actividad regular del proceso de formación intelectual [5], por lo que debe ser asumido desde las autoridades institucionales, y contar con el compromiso de las autoridades de la Escuela.

\section{REFERENCES}

[1] R. Aguila, B. Behnan, R. W. Burniske, C. Cerda, R. D. Valle, and M. Gonzalez, "Fase III: Integración de Tecnología y Currículum.," World Links, Washington, D.C., 2002.

[2] M. T. Huber, P. Hutchings, and R. Gale, "Integrative Learning for Liberal Education," in Integrative Learning, vol. 7, 4 vols., Asociation of America Colleges and Universities., 2005.

[3] S. Johnson, Integrative Learning, vol. 7, 4 vols. Asociation of America Colleges and Universities., 2005.

[4] Association of American Colleges and Universities (AAC\&U), "A statement on integrative learning," 2004. 
[5] D. Humphreys, "Why Integrative Learning? Why now?.," in Integrative Learning, vol. 7, 4 vols., Asociation of America Colleges and Universities, 2005, pp. 30-31.

[6] R. Vásquez and G. Urías, "Una Nueva Episteme de la Pedagogía Universitaria: La Integración Curricular de Los Procesos Académicos Universitarios," Práxis Educacional, vol. 8, no. 13, pp. 11-29, 2012.

[7] A. Camilloni, "Modalidades y proyectos de cambio curricular. Aportes para un cambio curricular en Argentina. Facultad de Medicina.," OMS, UBA-OPPS, 2001.

[8] A. Chaves, D. Hernández, J. León, Z. Pereira, and M. Vargas, "La integración curricular: Una experiencia en el primer nivel de diplomado de la carrera de Licenciatura en Pedagogía con énfasis en Educación Preescolar," Rev. Electrónica Educ., vol. 15, no. 2, pp. 6386, 2011.

[9] D. Perin, Curriculum and Pedagogy to Integrate Occupational and Academic Instruction in the Community College: Implications for Faculty Development, vol. 8. CCRC Brief, 2000.

[10] Reorganización curricular por ciclos: Referentes conceptuales y metodológicos. Colombia: Secretaría de Educación de Bogotá, 2012.

[11] C. Vasco, A. Bermúdez, H. Escobedo, J. C. Negret, and T. León, "El concepto de tópico generador," in El saber tiene sentido. una propuesta de integración curricular, 2000: CINEP.

[12] J. Negret, Proyectos y relatos. Herramientas para el diseño de proyectos pedagógicos. Bogotá: $\mathrm{H} \& \mathrm{G}, 2007$.

[13] L. Y. Aguilar, J. M. Carreño, A. Galeano, G. Preciado, and O. Espinoza, "Acerca del rediseño curricular por ciclos," Itinerario Educativo, vol. 55, pp. 213-237, 2010.
[14] R. Miller, "Integrate learning and Assessment," in Integrative Learning, vol. 7, 4 vols., Asociation of America Colleges and Universities., 2005.

[15] A. Kolmos, F. Fink, and L. Krogh, "The Aalborg Model - Problembased and Project-Organized Learning," in The Aalborg PBL model Progress, Diversity and Challenges, A. Kolmos, F. Fink, and L. Krogh, Eds. Aalborg: Aalborg University Press, 2004, pp. 9-18

[16] E. De Graaff and A. Kolmos, "Characteristics of problem-based learning.," Int. J. Eng. Educ., vol. 19, pp. 657-662, 2003.

[17] Universidad Nacional, "Modelo Pedagógico," 2007.

[18] M. . Sandoval and R. Cortés, "Proyecto: Análisis del impacto de proyectos desarrollados por los estudiantes de la Cátedra de Ingeniería de Sistemas de la UNA del 2006 al 2010," Universidad Nacional, 2011.

[19] T. Guskey, "Professional Development and Teacher Change," Teach. Teach. Theory Pr., vol. 8, pp. 381-391, 2002.

[20] T. Guskey, "What Makes Professional Development Effective?," Phi Delta Kappa Int., vol. 84, pp. 748-750, 2003.

[21] L. Esquivel, G. Induni, V. Madrigal, Z. Pereira, J. Solano, and W. Umaña, "Conformación de un equipo de investigadores: Una experiencia concreta," Revista Electrónica EDUCARE, vol. 6, pp. 193-204, 2004.

[22] M. Coto, S. Mora, and M. Lykke, "Design considerations for introducing PBL in Computer Engineering," Informática Clei 2012 Xxxviii Conf. Latinoam., pp. 1-10, 2012. 